\title{
Vanishing Wilson ratio as the hallmark of quantum spin-liquid models
}

\author{
P. Prelovšek, ${ }^{1,2}$ K. Morita, ${ }^{3}$ T. Tohyama $\odot,{ }^{3}$ and J. Herbrych $\oplus^{4}$ \\ ${ }^{1}$ Jožef Stefan Institute, SI-1000 Ljubljana, Slovenia \\ ${ }^{2}$ Faculty of Mathematics and Physics, University of Ljubljana, SI-1000 Ljubljana, Slovenia \\ ${ }^{3}$ Department of Applied Physics, Tokyo University of Science, Tokyo 125-8585, Japan \\ ${ }^{4}$ Department of Theoretical Physics, Faculty of Fundamental Problems of Technology, \\ Wroclaw University of Science and Technology, 50-370 Wroclaw, Poland
}

(Received 4 December 2019; accepted 13 March 2020; published 9 April 2020)

\begin{abstract}
We present numerical results for finite-temperature $T>0$ thermodynamic quantities, entropy $s(T)$, uniform susceptibility $\chi_{0}(T)$, and the Wilson ratio $R(T)$, for several isotropic $S=1 / 2$ extended Heisenberg models, which are prototype models for planar quantum spin liquids. We consider in this context the frustrated $J_{1}-J_{2}$ model on kagome, triangular, and square lattice, as well as the Heisenberg model on a triangular lattice with the ring exchange. Our analysis reveals that typically in the spin-liquid parameter regimes the low-temperature $s(T)$ remains considerable, while $\chi_{0}(T)$ is reduced consistent mostly with a triplet gap. This leads to vanishing $R(T \rightarrow 0)$, being the indication of a macroscopic number of singlets lying below triplet excitations. This is in contrast to the $J_{1}-J_{2}$ Heisenberg chain, where $R(T \rightarrow 0)$ either remains finite in the gapless regime, or the singlet and triplet gap are equal in the dimerized regime.
\end{abstract}

DOI: 10.1103/PhysRevResearch.2.023024

\section{INTRODUCTION}

Various frustrated $S=1 / 2$ Heisenberg models (HMs) have been the subject of intensive theoretical studies in last decades in connection with the possibility of a spin-liquid (SL) ground state (g.s.). These efforts have been recently strengthened by the discovery of several classes of insulating materials, revealing low-energy spin excitations behaving as a quantum SL without any magnetic order down to low temperatures (for reviews see [1-3]). Among isotropic $S=1 / 2$ twodimensional (2D) models, most numerical evidence for the SL g.s. accumulated for the antiferromagnetic (AFM) HM on the kagome lattice (KL) [4-10], but as well for $J_{1}-J_{2}$ $\mathrm{HM}$ on the square lattice (SQL) [11-18], $J_{1}-J_{2} \mathrm{HM}$ on the triangular lattice (TL) [19-24], and the HM on the TL with ring exchange $[25,26]$. While the character of the g.s. and its properties still offer several controversies and challenges, the finite-temperature $T>0$ behavior of several basic quantities is even less known. At least some of them have been already measured in experiments on SL materials and can thus serve as a test as to whether and to what extent actual materials can be accounted for by theoretical models.

Among measurable spin properties are thermodynamic quantities such as the uniform magnetic susceptibility $\chi_{0}(T)$, magnetic (contribution to) specific heat $C_{V}(T)$, and related spin entropy density $s(T)$. They are crucial to pinpointing the different characters and scenarios of SL behavior, in partic-

Published by the American Physical Society under the terms of the Creative Commons Attribution 4.0 International license. Further distribution of this work must maintain attribution to the author(s) and the published article's title, journal citation, and DOI. ular, whether materials follow gapped or gapless SL. These quantities are mostly extracted from experiments on KL systems, the prominent example being herbertsmithite [27-31], but are also related to compounds in the same class [32-37]. Another example is organic compounds where the relevant lattice is triangular [38-41], as well as the charge-density-wave system $1 \mathrm{~T}-\mathrm{TaS}_{2}$, recently established as a SL with composite $S=1 / 2$ spins on TL [42-45]. The basic spin exchange scale in most of these systems is modest, and as a consequence, the whole $T$ range is experimentally accessible, which allows for the test of the whole range of spin excitations. Nevertheless, it should be noted that properties at lowest $T$ might be influenced by additional mechanisms such as DzyaloshinskiiMoriya interaction [46-48], interlayer couplings, and random effects [49].

It has been rather well established that with elaborate exact-diagonalization (ED) and series-expansion studies of the HM with nearest-neighbor (n.n.) exchange on KL [4-6,8,50,51], lowest excitations are singlets dominating over the triplet excitations, for which most ED studies reveal a finite spin (triplet) gap $\Delta_{t}>0$, although there are numerical indications also for the gapless scenario [52,53]. It has been recently shown [54] that the same scenario can be traced via the temperature-dependent Wilson ratio $R(T)$ in $J_{1}-J_{2} \mathrm{HM}$ on TL including the next-nearest-neighbor (n.n.n.) exchange $J_{2}>0$ in the regime where the SL g.s. is expected [19-22]. This is in contrast with the triplet $(S=1)$ magnon excitation being the lowest excitations in an ordered AFM. It is also qualitatively different from the scenario for the basic onedimensional (1D) HM with gapless spinon excitations.

In the following we present numerical results for $s(T)$, $\chi_{0}(T)$, and $R(T)$, which reveal that the vanishing $R(T \rightarrow$ $0)$ is a quite generic property of a wide class of isotropic 2D Heisenberg models in their range of (presumable) SL 
parameter regimes. In this context we generalize previous numerical $T>0$ studies of $\mathrm{HM}$ on $\mathrm{KL}[55,56]$ to include also the n.n.n. exchange $J_{2} \neq 0$ and upgrade results for the $J_{1}-J_{2}$ HM on TL [24], now studying also the HM on TL with the ring exchange, as well as another standard model of SL, i.e., frustrated $J_{1}-J_{2} \mathrm{HM}$ on SQL. Results in the SL regimes confirm singlets as dominating low-energy excitations. For comparison, we present results also for $1 \mathrm{D} J_{1}-J_{2}$ Heisenberg chains, which serve as the reference, depending on $J_{2} / J_{1}$, either for the gapless spinon Fermi-surface (SFS) and valence-bond (VB) solid scenarios. Still, we show that results appear (as expected) qualitatively different from considered $2 \mathrm{D}$ models.

Investigated models have their particular features and challenges; nevertheless, our results on thermodynamic properties reveal quite universal properties in their (presumable) SL regimes which also put restrictions on the SL scenarios explaining their low- $T$ behavior. In particular, very attractive scenarios of gapless SL with SFS excitations require finite g.s. Wilson ratio $R_{0}=R(T \rightarrow 0)>0$. The latter is realized in 1D HM but does not appear to be the case in planar models. Observed enhanced low- $T$ entropy $s(T)$ and related vanishing $R_{0}=0$ demonstrate the dominant role of singlet excitations over the triplet excitations [50,57] but still offer several possibilities. While it is hard to exclude the scenario of VB solids (crystal) with broken translational symmetry [6,50], it is more likely that the g.s. in the SL regime does not break the translation symmetry and all correlations are short-ranged, i.e., revealing a scenario of VB (or dimer) liquid. On the other hand, it is very possible that considered models might not be enough to represent the SL real materials, in particular, not in their low- $T$ regime.

The paper is organized as follows: In Sec. II we introduce a $T$-dependent Wilson ratio $R(T)$ and comment on different scenarios for its low- $T$ behavior. In Sec. III we present numerical methods used to evaluate thermodynamic quantities but also lowest spin excitations in 1D and 2D models. As a test of methods as well as of concepts we present in Sec. IV results for a $1 \mathrm{D} J_{1}-J_{2}$ Heisenberg chain. The central results for various 2D frustrated HM models are presented and analyzed in Sec. V and summarized in Sec. VI.

\section{TEMPERATURE-DEPENDENT WILSON RATIO}

Besides thermodynamic quantities, uniform magnetic susceptibility $\chi_{0}(T)$ and the entropy density $s(T)$, together with related specific heat $C_{V}(T)=T d s / d T$, it is informative to extract also their quotient in the form of temperature-dependent Wilson ratio $R(T)$, defined as [54,58]

$$
R(T)=\frac{4 \pi^{2} T \chi_{0}(T)}{3 s(T)},
$$

being a dimensionless quantity assuming (theoretical) units $k_{B}=g \mu_{B}=1$. It should be remembered that the standard quantity is the (zero-temperature) Wilson ratio as $R_{W}=4 \pi^{2} \chi_{0}^{0} /(3 \gamma)$, where $\chi_{0}^{0}=\chi_{0}(T=0)$ and $\gamma=$ $\lim _{T \rightarrow 0}\left[C_{V} / T\right] . R_{W}$ has its usual application and meaning in the theory of Fermi liquids and metals, as well as in gapless spin systems [59]. We note that in normal Fermi-liquidlike systems where $s=C_{V}=\gamma T$, the definition, Eq. (1), coincides at $T \rightarrow 0$ with the standard $R_{W}$. Although at low $T$ (in most interesting cases) both $s(T)$ and $C_{V}(T)$ have the same functional $T$ dependence, it is more convenient to employ in Eq. (1) the entropy density $s(T)$ being a monotonously increasing function.

It should be also pointed out that $R(T)$ is a direct measure of the ratio of the density of excitations with finite $z$ component of total spin $S_{\text {tot }}^{z} \neq 0$ relative to the density of all (spin) excitations, including $S_{\text {tot }}^{z}=0$, as measured by $s(T)$. To make this point evident, we note that $\chi_{0}(T)=\left\langle\left(S_{z}^{\text {tot }}\right)^{2}\right\rangle /(N T)$, where $N$ is the number of lattice sites, so that

$$
R=\frac{4 \pi^{2}\left\langle\left(S_{\mathrm{tot}}^{z}\right)^{2}\right\rangle}{3 N s} .
$$

From the above expression it is also follows that $R(T)$ has a well-defined high- $T$ limit which is for isotropic $S=1 / 2 \mathrm{HM}$ $R(T \rightarrow \infty)=\pi^{2} /(3 \ln 2)=4.746$.

Moreover, $R_{0} \equiv R(T \rightarrow 0)$ can differentiate between distinct scenarios:

(a) In the case of magnetic long-range order (LRO), e.g., for $\mathrm{AFM}$ in $\mathrm{HM}$ on SQL, at $T \rightarrow 0$ one expects, in 2D isotropic $\mathrm{HM}, \chi_{0}(T \rightarrow 0)=\chi_{0}^{0}>0$ (where the finite value can be interpreted as the contribution of spin fluctuations transverse to the g.s. magnetic order), whereas effective magnon excitations lead to $s \propto T^{2}$ [60], so that $R_{0} \propto 1 / T \rightarrow$ $\infty$

(b) In a gapless SL with large SFS one would expect Fermi-liquid-like finite $R_{0} \sim 1$ [2,41,44]. The evident case for such a scenario, as a reference for consideration later on, is the simple Heisenberg chain where $R_{0}=2$ [61], in contrast to the value $R_{0}=1$ for noninteracting Fermi systems.

(c) Vanishing $R_{0} \rightarrow 0$, or more restricted from Eq. (1) $R_{0} \propto T^{\eta}$ with $\eta \geqslant 1$, would indicate that low-energy singlet excitation dominates over the triplet ones [2,50,51]. In the following we find numerical evidence that this appears to be the case in the SL parameter regime of considered 2D frustrated isotropic HM.

Within the last scenario one should still differentiate different possibilities with respect to gapless spin systems or systems with the gap. One option for SL is that both singlet and triplet excitations are gapped, but the effective triplet gap is larger $\Delta_{t}>\Delta_{s}$ (in the limit of large systems $N \rightarrow \infty$ ) which would lead (in a simplest approximation) to $R_{0} \propto$ $T^{\eta} \exp \left[-\left(\Delta_{t}-\Delta_{s}\right) / T\right] \rightarrow 0$. A more delicate case could be when $\Delta_{t}=\Delta_{s}=\Delta$. Then Eq. (1) offers several scenarios with, e.g., $R(T<\Delta) \propto T^{\eta}$. Such a situation appears, e.g., for $1 \mathrm{D}$ chain $J_{1}-J_{2}$ models around the Mazumdar-Ghosh point $J_{2} / J_{1}=0.5$. Since $s(T)$ measures both singlet and triplet excitations (as well as higher $S_{\text {tot }}>1$ ), the possible case $\Delta_{s}>$ $\Delta_{t}$ should be similar to the previous scenario.

When classifying options for $T \rightarrow 0$ we should also consider the possibility of a VB solid (crystal), i.e., the g.s. with broken translational symmetry. In finite systems (with short-range spin correlations) the signature of the VB solid should be the degenerate or (due to finite-size effects) nearly degenerate g.s. with degeneracy $N_{d}>1$. This should be reflected in a finite g.s. entropy for finite systems with $N$ sites:

$$
s_{0} \equiv s(T \rightarrow 0)=\frac{1}{N} \ln N_{d}
$$


Such remnants $s_{0}>0$ do not contribute to $C_{V}(T)$ and moreover vanish in the limit $N \rightarrow \infty$. A clear VB solid case is 1D $J_{1}-J_{2} \mathrm{HM}$ in the dimerized regime where $N_{d}=2$. It then makes sense to consider in the evaluation of $R(T)$, Eq. (1), besides the full $s(T)$ and also the reduced one, $\tilde{s}=s-s_{0}$. Still, it is not always straightforward to fix proper $N_{d}$ in finite-size systems.

\section{METHODS}

We calculate entropy density $s(T)$, uniform susceptibility $\chi_{0}(T)$, and via Eq. (1) the Wilson ratio $R(T)$, using the finite-temperature Lanczos method (FTLM) [58,62], previously used in numerous studies of $T>0$ static and dynamical properties in various models of correlated electrons [63]. Since in the case of considered thermodynamic quantities only conserved quantities are involved, in particular the Hamiltonian $H$ and $S_{\text {tot }}^{z}$, the memory and CPU time requirement for a given system size $N$ are essentially those of the Lanczos procedure for the g.s., provided that we scan over all (different) symmetry sectors $S_{\text {tot }}^{z}$ and the wave vector $\mathbf{q}$ due to translational symmetry and periodic boundary conditions (p.b.c.), in the case of the code with translational symmetry. A modest additional sampling $N_{s}$ over initial wave functions is then used. Limitations of the present method are given by the size of the many-body Hilbert space with $N_{s t}$ basis states, which can be handled efficiently within the FTLM, restricting in our study lattice sizes to $N \leqslant 36$. In the following we use two FTLM codes for the considered models:

(a) To calculate the largest systems with $N=36$ sites for the 2D TL, KL as well as SQL $J_{1}-J_{2} \mathrm{HM}$ with $N_{s t} \sim 10^{10}$, we develop a code that equips a technique to save the memory for the Hamiltonian by dividing $H$ into two subsystems. In addition, to improve the accuracy, we use a replaced FTLM technique [64].

(b) The code for more modest computers takes into account translational symmetry and thus is able to reach $N_{s t}<10^{7}$ and sizes $N \leqslant 30$, used for the 1D HM chain and the TL with ring exchange.

When discussing the accuracy of FTLM results, we have to distinguish results for given systems from finite-size effects due to restricted $N$. The central quantity evaluated is the grand-canonical sum $[58,62]$,

$$
Z(T)=\operatorname{Tr} \exp \left[-\left(H-E_{0}\right) / T\right],
$$

where $E_{0}$ is the g.s. energy. For reachable systems FTLM provides accurate results, provided that we use modest random sampling $N_{s} \leqslant 30$ over (random) initial wave functions. This is particularly important to get a correct low- $T$ limit, i.e., $Z(T=0)=1$ in the case of nondegenerate g.s. [64]. The main restriction of FTLM results are, however, reachable $N$ and related finite-size effects most pronounced at $T \rightarrow 0$ :

(a) In isotropic HM with $T \rightarrow 0$ LRO (in dimension $D \geqslant 2$ ), or long-range spin correlations in $1 \mathrm{D}$, spin excitations are gapless in the thermodynamic limit. Such a case is correlated with finite-size effects in evaluated quantities. One can expect that results reach the $N \rightarrow \infty$ validity only for $Z>Z^{*}=Z\left(T_{f s}\right) \gg 1$. Since $Z$ is intimately related to entropy,

$$
s=\frac{1}{N}\left(\ln Z+\frac{\langle H\rangle-E_{0}}{T}\right),
$$

the criterion for $T_{f s}$ can be the smallest value for $s$. Actually, in reached systems $N \sim 36$ we get an estimate $s\left(T_{f s}\right) \sim 0.07-$ 0.1 (see, e.g., the finite-size analysis in [56]). In such systems $s(T)$ and $\chi_{0}(T)$ results at $T<T_{f s}$ are dominated by finite-size effects and are not representative for $N \rightarrow \infty$. In any case, due to frustration and consequently enhanced $s\left(T \ll J_{1}\right)$ in SL models, FTLM generally allows a lower $T_{f s}$ to be reached, e.g., while for HM on an unfrustrated SQL (even at the largest $N, N=36) T_{f s} \sim 0.4 J_{1}[58,63]$, SL models allow for considerably lower $T_{f s} \leqslant 0.1[24,56]$.

(b) For systems with only short-range spin correlations, one can reach a situation where the spin correlation length (even at $T \rightarrow 0$ ) is shorter that the system length, $\xi \leqslant L$. In such a case, FTLM has no obvious restrictions even at $T \rightarrow 0$, so $T_{f s} \sim 0$. This can be the situation for gapped SL, including some examples discussed further on.

Besides thermodynamic quantities, it is also instructive to directly monitor the lowest excited states and their character. For the largest 2D $N=36$ systems, excited states are obtained within ED (without translational symmetry) by eliminating Lanczos ghost states while comparing results for different number of Lanczos steps. For TL with ring exchange we employ ED results of systems with $N=28$ and evaluate the lowest (singlet and triplet) energies in different $\mathbf{q}$ sectors.

In 1D models we use also density matrix renormalization group (DMRG) method to investigate the $J_{1}-J_{2} \mathrm{HM}$ with open boundary conditions (o.b.c.). The method allows for accurate computation of the $S_{\text {tot }}^{z}=0$ g.s. and in the same way also first excited triplet state with $S_{\text {tot }}^{z}=1$. In order to get also excited (singlet) states within the $S_{\text {tot }}^{z}=0$ sector, we evaluate the g.s. eigenfunction $\left|\psi_{0}\right\rangle$ and then construct an effective Hamiltonian for the excited states $H_{1}=H-E_{0}\left|\psi_{0}\right\rangle\left\langle\psi_{0}\right|$ [17] and then repeat the standard DMRG algorithm for $H_{1}$. The requirement of orthogonality is, however, difficult to meet for excited states which are (due to o.b.c.) edge states, e.g., as within a $1 \mathrm{D}$ dimerized regime.

\section{ONE-DIMENSIONAL HEISENBERG MODEL}

We consider first the $1 \mathrm{D} J_{1}-J_{2} \mathrm{HM}$, which can serve as the reference for further discussion of $2 \mathrm{D}$ HM results. The AFM isotropic $S=1 / 2 J_{1}-J_{2} \mathrm{HM}$ is given by

$$
H=\sum_{i}\left[J_{1} \mathbf{S}_{i} \cdot \mathbf{S}_{i+1}+J_{2} \mathbf{S}_{i} \cdot \mathbf{S}_{i+2}\right],
$$

where we later put $J_{1}=J=1$ as the unit of energy. We will investigate with FTLM only the $J_{2} \geqslant 0$ case on systems of finite length $N$ with p.b.c. Thermodynamic properties are well known and understood for a simple $J_{2}=0$ Heisenberg chain [61], as well the g.s. and the triplet excited state for the frustrated chain with $J_{2}>0$ [65]. Beyond critical $J_{2}>J_{2}^{*} \sim$ 0.241 , the g.s. is dimerized $\left(N_{d}=2\right)$ in the thermodynamic limit [65]. At the same time, the lowest excited states are degenerate triplets and singlets with the gap $\Delta_{t}=\Delta_{s}$, consistent with the unbound spinons as elementary excitations. 

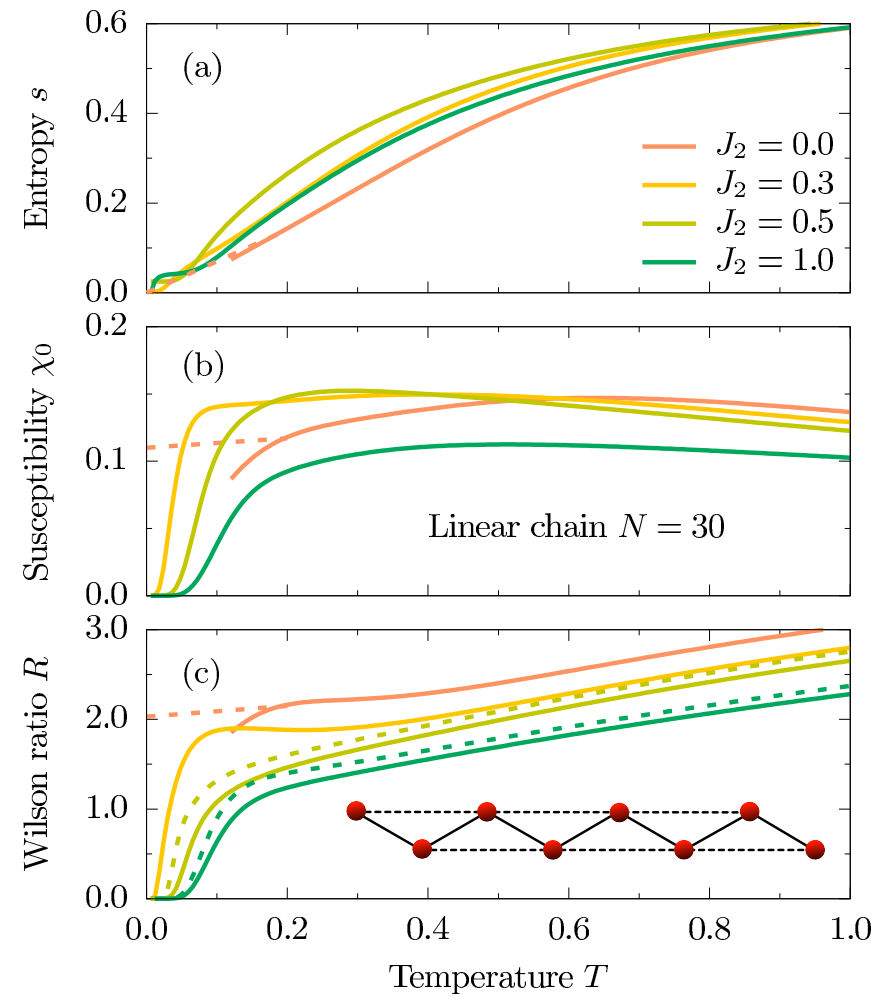

FIG. 1. Results in the $J_{1}-J_{2}$ Heisenberg chain for (a) entropy $s(T)$, (b) susceptibility $\chi_{0}(T)$, and (c) Wilson ratio $R(T)$, as obtained via FTLM on $N=30$ sites for different $J_{2}=0.0-1.0$. The dashed lines at $J_{2}=0$ represent the extension to $N \rightarrow \infty$, while for $J_{2}=$ $0.2,0.3$ they denote modified $R(T)$ evaluated with reduced $\tilde{s}(T)$. The inset in (c) represents a sketch of $J_{1}$ (solid line) and $J_{2}$ (dashed line) in a $1 \mathrm{D}$ Heisenberg chain.

Numerical results for $s(T), \chi_{0}(T)$, and finally $R(T)$, as obtained on a system with $N=30$ sites, are presented in Fig. 1 for different $0 \leqslant J_{2} \leqslant 1$ :

(a) For the simple $J_{2}=0$ chain we get $s(T) \sim \gamma T$ in a very broad range $T<0.6$. Finite-size effects are most pronounced in this case, so that below $T<T_{f s} \sim 0.2$ we get $s<0.1$ and finite-size effects prevent any further firm conclusions. Still, for $T>T_{f s}$, numerical results are consistent with analytical and previous numerical results, in particular with the known limit $R_{0}=2$ [61]. Moreover, it is remarkable that $R(T)$ is nearly constant in a wide range $T<0.6$.

(b) The gap becomes pronounced for the Mazumdar-Ghosh point $J_{2}=0.5$ and even more for $J_{2}=1.0$ (where $\Delta_{t} \sim$ 0.25 [65]). In the gapped case FTLM finite-size effects are less pronounced, and one can expect $T_{f s} \rightarrow 0$. In fact, for $J_{2}=0.5$ and $J_{2}=1.0$, results appear size-independent for $N=30$, apart from the dimerization degeneracy $N_{d}=2$, leading via Eq. (3) to $s_{0}>0$. The latter has influence on the $R(T \sim 0)$, so we present in Fig. 1 also the result taking into account subtracted $\tilde{s}(T)$. In both analyses the behavior is consistent with $R_{0}=0$. For the $J_{2}=0.5$ and $J_{2}=1.0$, modified results are still consistent with vanishing $R(T<\Delta) \propto T^{\eta}$ with $\eta \geqslant$ 1 , but this behavior remains to be clarified. For the marginal case $J_{2}=0.3 \sim J_{2}^{*}$, the behavior of all quantities is similar to $J_{2}=0$, except that we find larger $\gamma$ and consequently also smaller $T_{f s}$.
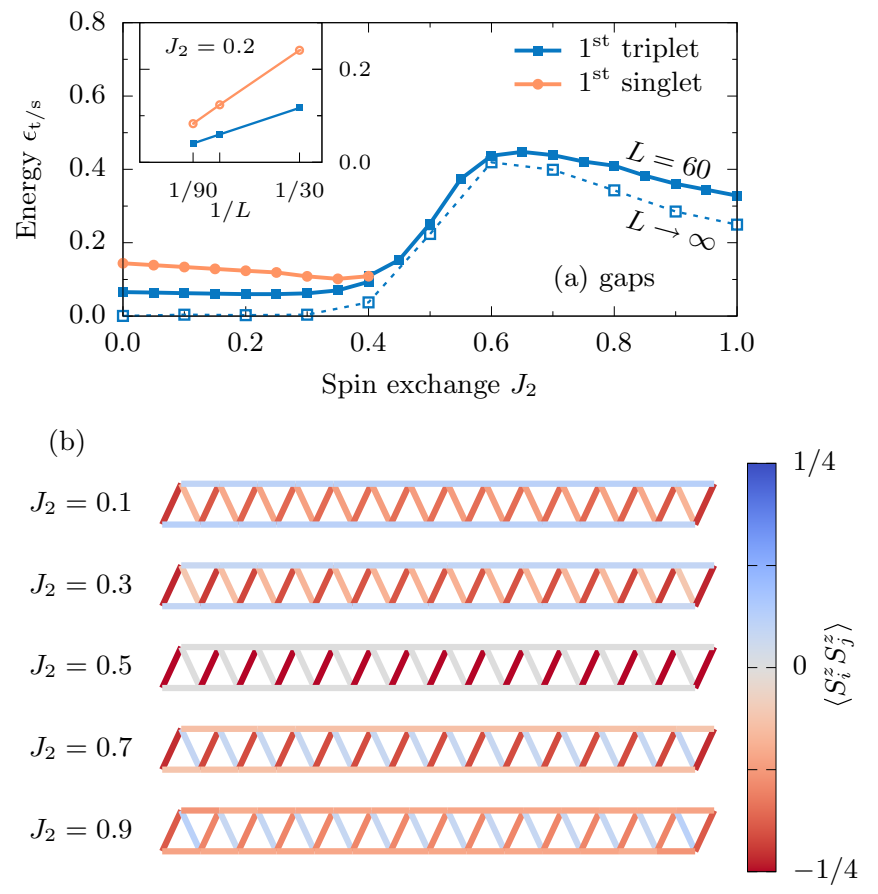

FIG. 2. (a) Lowest triplet $\epsilon_{t}$ and singlet $\epsilon_{s}$ excitations vs $J_{2}$, as obtained via DMRG in the chain of $N=60$ sites, with the inset showing the scaling of $\epsilon_{t / s}$ vs $1 / N$ for $J_{2}=0.2$. (b) Corresponding g.s. spin correlations $\left\langle S_{i}^{z} S_{j}^{z}\right\rangle$ on particular bonds.

It is instructive to investigate in connection with finite-size effects also lowest triplet and singlet excitations in the model. While triplet excitations have been studied in detail using DMRG already in Ref. [65], to establish singlet excitations requires more care, see Sec. III. In Fig. 2(a) we present the DMRG (with o.b.c.) $N=60$ result for excitations: the lowest triplet $\epsilon_{t}$ and lowest singlet $\epsilon_{s}$ vs $J_{2}$, together (as the inset) with their $1 / N$ scaling in the gapless regime $J_{2}=0.2<$ $J_{2}^{*}$. Due to o.b.c., DMRG is unable to properly resolve the dimerized partner of g.s., since it represents an open chain with excited edge states. Hence, we present in Fig. 2(a) the first singlet excited state only for $J_{2} \leqslant 0.4$. Still, the DMRG results confirm that no other singlet is stable below the triplet for $J_{2}>J_{2}^{*}$, unlike what is seen later on in 2D SL models.

In Fig. 2(b) we display also DMRG results for g.s. bond spin correlations $\left\langle S_{i}^{z} S_{j}^{z}\right\rangle$. It is also apparent that for $J_{2}>J_{2}^{*}$ the g.s. is dimerized (in n.n. bond correlations), whereby the particular case is $J_{2}=0.5$ with alternating n.n. correlations $\left\langle S_{i}^{z} S_{j}^{z}\right\rangle=-1 / 4$ and 0 . Stronger correlations remain for AFM in the whole $J_{2}>J_{2}^{*}$, while it is easy to recognize the change of character of weaker bonds from AFM correlations for $J_{c}^{*}<$ $J_{2}<0.5$, to ferromagnetic ones for $J_{2}>0.5$.

\section{PLANAR FRUSTRATED HEISENBERG MODELS}

\section{A. $J_{1}-J_{2}$ Heisenberg model on kagome lattice}

$\mathrm{HM}$ on $\mathrm{KL}$ is the prototype model for the existence of SL in planar models. It has been the subject of numerous studies, devoted mostly to the g.s. using ED [4,5,8,51], series expansion [6], DMRG [7,10,66], and variational methods [9,52]. We 

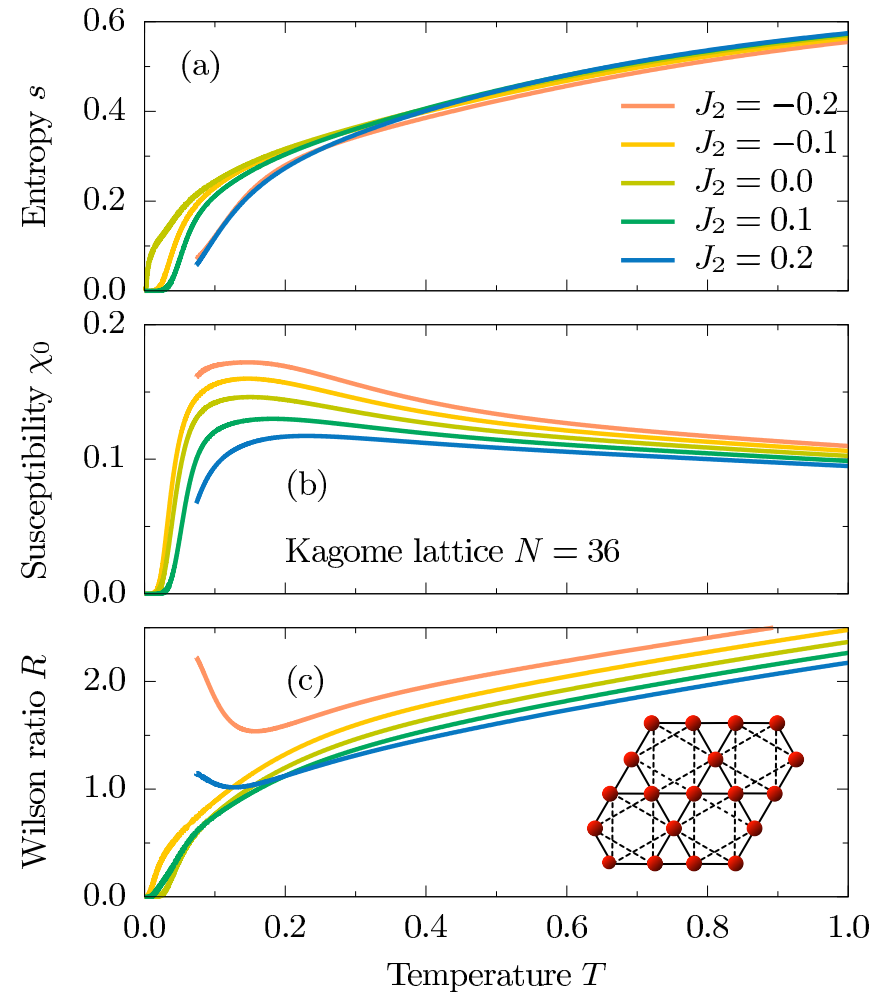

FIG. 3. $s(T), \chi_{0}(T)$, and $R(T)$ within the $J_{1}-J_{2} \mathrm{HM}$ on KL, obtained via FTLM on $N=36$ sites, for different $\left|J_{2}\right| \leqslant 0.2$. The inset in (c) represents a sketch of the $J_{1}$ (solid line) and $J_{2}$ (broken line) connections in KL.

consider here the extended model with p.b.c., involving also the n.n.n. exchange $J_{2}$, as shown in the inset of Fig. 3(c),

$$
H=J_{1} \sum_{\langle i j\rangle} \mathbf{S}_{i} \cdot \mathbf{S}_{j}+J_{2} \sum_{\langle\langle i l\rangle\rangle} \mathbf{S}_{i} \cdot \mathbf{S}_{l},
$$

whereby the role of $J_{2}>0$, as well as $J_{2}<0$, is to reestablish the magnetic LRO [67]. The basic HM on KL has been the clearest case for a dominant role of low-lying singlet excitations over the triplet ones [6,50,51]. The latter fact and related large entropy, persistent at low $T \ll 1$, has been well captured within block-spin $[4,5,68]$ and recently within related reduced-basis approaches [54], whereby singlet excitations can be attributed to chiral fluctuations, distinct from (higher-energy) triplet excitations.

Thermodynamic quantities for the basic $J_{2}=0 \mathrm{HM}$ on KL have been calculated via FTLM previously [56] up to the size $N=42$. Here we extend the study, evaluating via FTLM also for $J_{2} \neq 0$ for $N=36$. Results in Fig. 3 reveal that increasing $\left|J_{2}\right|>0$ strongly suppresses $s\left(T \ll J_{1}\right)$ while leaving $\chi_{0}(T)$ less affected (at least for $T>T_{f s}$ ). Results for $J_{2}= \pm 0.2$ indicate divergent $R_{0} \rightarrow \infty$, consistent with the emergent magnetic LRO [54,67]. On the other hand, at $\left|J_{2}\right| \leqslant 0.1$ the behavior of $\chi_{0}(T)$ and $s(T)$ are consistent with the finite triplet gap $\Delta_{t} \sim 0.15$ and a smaller or even vanishing singlet gap $\Delta_{s}<\Delta_{t}$.

Results in Fig. 3 are quite robust against finite-size effects, in particular, in the presumable SL regime. To substantiate this we show in Fig. 4(a) $R(T)$ in a low- $T$ regime for the $\mathrm{KL}$ at
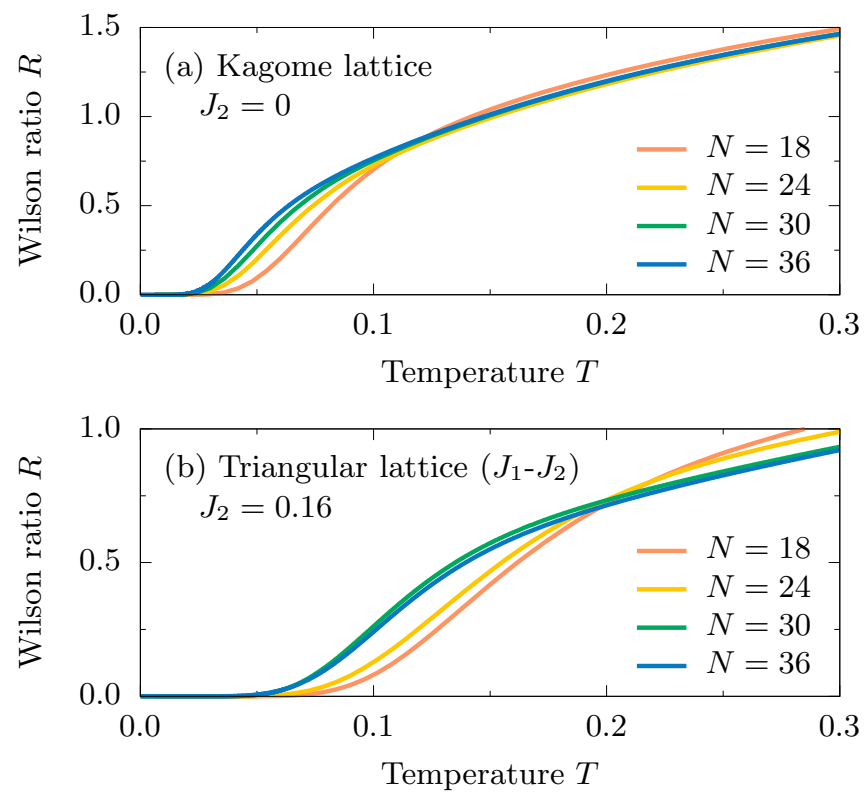

FIG. 4. Finite-size comparison of $R(T)$ within the SL regimes for (a) basic KL model with $J_{2}=0$ and (b) $J_{1}-J_{2}$ model on TL. Results are obtained via FTLM on lattices with $N=18-36$ sites.

$J_{2}=0$, as obtained via FTLM on lattices of quite different sizes $N=18-36$. It should be stressed that we do not pretend to perform a proper finite-size scaling, since considered lattices are not just of different sizes but also of different shapes (due to the requirement of p.b.c.), e.g., lacking some (rotational) symmetries, etc. Still, results for $R(T)$ in Fig. 4(a) [as well as for the TL on Fig. 4(b), discussed further on] reveal quite systematic evolution of $R(T)$ consistent with vanishing $R(T \rightarrow 0)$.

The transition from the singlet-dominated SL regime to the phases with magnetic LRO can be monitored also via low-lying levels in considered systems. In Fig. 5 we present the evolution of excitation energies for the lowest lying triplet $\epsilon_{t}$ as well as several low-lying excited singlets $\epsilon_{s, i}(i=$ $1,2, \cdots, 6$ ), as obtained via ED on $N=36$ sites, and in part for $N=32$ sites for the HM on TL with ring exchange. It should be pointed out that we monitor only nondegenerate excited states, whereby in general the degeneracy is present and depends on a particular lattice and related p.b.c. The level evolution, plotted vs $J_{2}$ (or $J_{r}$ discussed later on), serves primarily as another test where one can expect SL with a macroscopic number (in the limit $N \rightarrow \infty$ ) of singlet excitations below the triplet ones, but also to locate transitions between different regimes.

In Fig. 5(a) the level scheme for KL is consistent with the previous ED studies of the $\left(J_{2}=0\right) \mathrm{KL}$ model $[6,50,51]$ which reveal a massive density of singlet levels with $\epsilon_{s} \sim 0$ below the lowest triplet one $\epsilon_{t}$. Introducing $\left|J_{2}\right|>0$ reduces the degeneracy and might lead to $\Delta_{s}>0$, even in the $N \rightarrow \infty$ limit. Still, a large density of singlet levels appears below the triplet in a wide (SL) range $J_{2}^{c 1}<J_{2}<J_{2}^{c 2}$, where $J_{2}^{c 1} \sim$ $-0.1, J_{c}^{c 2} \sim 0.1$ from Fig. 5(a) and we define $J_{2}^{c 1, c 2}$ with the crossing of (all) lowest $\epsilon_{s, 1-6}<\epsilon_{t}$. We note that marginal 

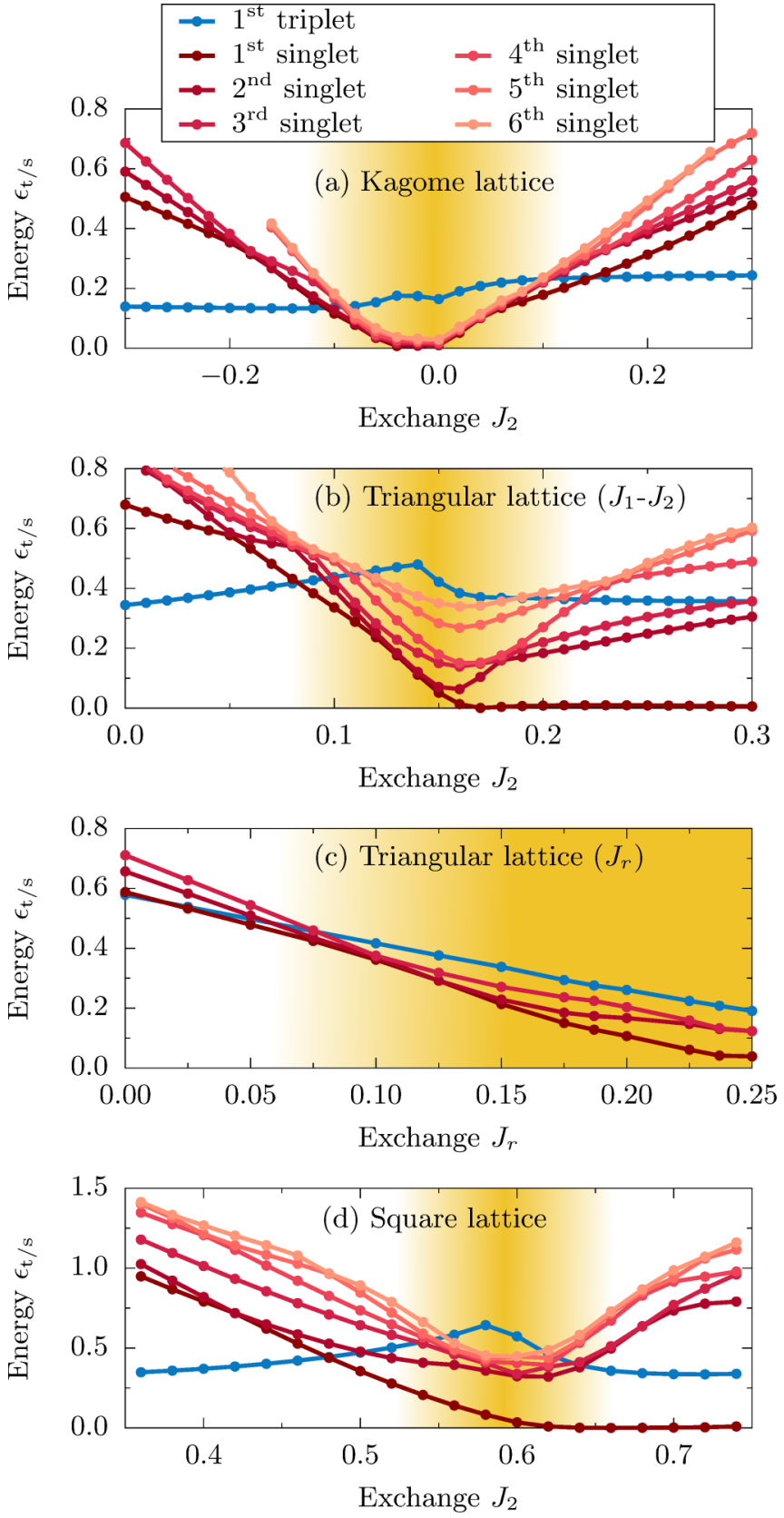

FIG. 5. Lowest triplet excitation $\epsilon_{t}$ and (nondegenerate) singlet excitations $\epsilon_{s, i}(i=1,2, \ldots, 6)$ vs $J_{2}$ for different planar $J_{1}-J_{2} \mathrm{HM}$ models on: (a) KL, (b) TL, and (d) SQL, as obtained with ED on $N=36$ sites, and (c) $\epsilon_{t}$ and $\epsilon_{s, i=1,2,3}$ vs $J_{r}$ on TL with ring exchange, obtained on $N=28$ sites.

$J_{2}^{c 1, c 2}$ are consistent with Fig. 3 , where $J_{2}= \pm 0.2$ already reveal magnetic LRO with $R_{0} \rightarrow \infty$.

\section{B. $J_{1}-J_{2}$ Heisenberg model on triangular lattice}

While numerical studies for the basic $\left(J_{2}=0\right) \mathrm{HM}$ on TL [69-71] confirm magnetic LRO with moments pointing into $120^{\circ}$-angle directions, modest additional frustration with $J_{2}>0$ allows for the possibility of SL g.s., with evidence for either gapless [19] or gapped SL [20-23] in the intermediate regime $J_{2} \sim 0.15$. Beyond that, for the $J_{2}>0.2$ stripe AFM is
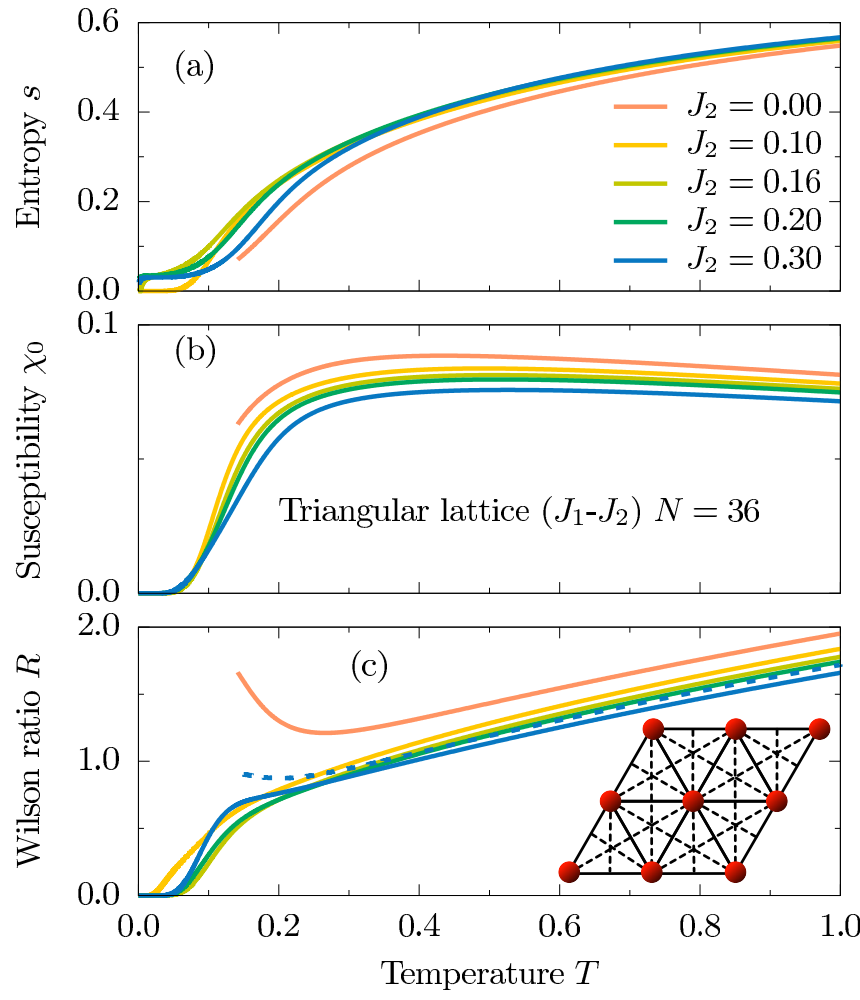

FIG. 6. $s(T), \chi_{0}(T)$, and $R(T)$ within $J_{1}-J_{2} \mathrm{HM}$ on TL, obtained via FTLM on $N=36$ sites for different $J_{2} \leqslant 0.3$. The dashed line for $J_{2}=0.3$ represents results using reduced $\tilde{s}(T)$. The inset in (c) represents a sketch of the $J_{1}$ (solid line) and $J_{2}$ (broken line) connections in TL.

expected. Thermodynamic (and some dynamic) quantities for the $J_{1}-J_{2} \mathrm{HM}$, Eq. (7), on TL have been recently calculated using FTLM [24] up to $N=30$ sites and employing the reduced-basis approach [54], whereby the similarity of $s(T)$, $\chi_{0}(T)$, and $R(T)$ with the basic HM on KL in the SL regime in both models has been attributed to chiral fluctuations dominating low- $T$ excitations.

Here we upgrade previous FTLM studies with the calculation of $J_{1}-J_{2} \mathrm{HM}$ on TL with $N=36$ sites. Results in Fig. 6 are qualitatively consistent with previous ones for $N=30$ [24], but due to larger size and consequently smaller $T_{f s}$, results are reliable to smaller entropy $s(T)$ and more evidently reveal diverging $R(T)$ below $T \sim 0.2$ for $J_{2} \sim 0$, where the g.s. possesses magnetic LRO. A similar behavior is expected for $J_{2}>$ 0.2 where the stripe AFM g.s. has been established [19]. In the reachable system $N=36$ the upturn of $R(T)$ is partly masked by finite-size $s_{0}>0$, Eq. (3), due to the degeneracy $N_{d}>1$ of striped magnetic LRO, evident in Fig. 6(a) at $J_{2}=0.2$ and 0.3 . Taking into account in Eq. (1) the reduced $\tilde{s}=s-s_{0}$, we obtain for $J_{2}=0.3$ again the indication for the upturn of $R(T)$ consistent with g.s. magnetic LRO. Still, in the most important intermediate regime $0.1<J_{2}<0.2$ the increase of $s_{0}$ and at the same time fast decrease of $\chi_{0}(T \rightarrow 0)$ (indicating a finite triplet gap $\Delta_{t}>0$ ) leads to vanishing $R_{0}=0$ and is consistent with interpretation with the SL g.s. [19-23].

Again, in this intermediate regime results are most robust against finite-size effects. In Fig. 4 we display the results for $R(T)$ for particular $J_{2}=0.16$, as obtained on quite different 
sizes $N=18-36$, whereby all presented lattices are not optimal with respect to lattice symmetries. Nevertheless, low$T$ variation of $R(T)$ appears at least qualitatively consistent for all $N$.

In Fig. 5(b) we plot the corresponding evolution of excitations vs $J_{2}$ as obtained with ED on an $N=36$ lattice. The triplet gap apparently remains substantial, i.e., $\epsilon_{t}>0.38$ for considered $N$ in the whole range of $J_{2}<0.3$. Still, singlet excitations $\epsilon_{s, 1-6}$ all cross $\epsilon_{t}$ for small $J_{2} \sim 0.1$. This effectively leads to g.s. level crossing $\epsilon_{s, 1}=0$ at $J_{2} \sim 0.17$, exchanging the character of the g.s. into a striped AFM. But most importantly, in the intermediate range $0.1<J_{2}<0.17$, which should be the relevant SL regime, singlet-excitation collapse is consistent with the conclusions from thermodynamics in Fig. 6 and $R_{0}=0$. It should be, however, acknowledged that the singlet collapse is not as pronounced as for basic $\left(J_{2} \sim 0\right)$ $\mathrm{HM}$ on KL in Fig. 5(a).

\section{Heisenberg model with ring exchange on triangular lattice}

While $J_{1}-J_{2}$ HM on TL is conceptually simple, it is less obvious to justify in connection with experiments and with more basic models. The organic SL materials [38-41] and $1 \mathrm{~T}-\mathrm{TaS}_{2}$ [42-45] are closer to the metal-insulator transition where simple $S=1 / 2$ n.n. HM is presumably not enough. Assuming as the starting point the single-band Hubbard model on the insulator side of the Mott transition $U>U_{c}$, the lowest correction to the n.n. HM comes in the form of the ringexchange term $[25,26,72,73]$,

$$
H=J \sum_{\langle i j\rangle} \mathbf{S}_{i} \cdot \mathbf{S}_{j}+H_{r},
$$

with

$$
\begin{aligned}
H_{r}= & \frac{J_{r}}{2} \sum_{\langle i j k l\rangle}\left(P_{i j k l}+P_{l k j i}\right) \sim J_{r} \sum_{\langle i j k l\rangle}\left[\left(\mathbf{S}_{i} \cdot \mathbf{S}_{j}\right)\left(\mathbf{S}_{k} \cdot \mathbf{S}_{l}\right)\right. \\
& \left.+\left(\mathbf{S}_{i} \cdot \mathbf{S}_{l}\right)\left(\mathbf{S}_{j} \cdot \mathbf{S}_{k}\right)-\left(\mathbf{S}_{i} \cdot \mathbf{S}_{k}\right)\left(\mathbf{S}_{j} \cdot \mathbf{S}_{l}\right)\right],
\end{aligned}
$$

where $\langle i j k l\rangle$ are taken over different four-cycles on TL, as shown in the inset of Fig. 7(c). $H_{r}$, Eq. (9), has been confirmed as the leading correction in the numerical study of the halffilled Hubbard model [72] in the insulating regime where $J_{r} \sim 80 t^{4} / U^{3} \sim\left(20 t^{2} / U^{2}\right) J<0.2 J$ [73], taking into account that the Mott insulator on TL requires $U>U_{c} \sim 8 t-10 t$ and $J \sim 4 t^{2} / U$. It should be also mentioned that in Eq. (9) in our numerical study we neglect the (small) corrections to the n.n. exchange term and corresponding $J$, which emerge in the same order of the $t / U$ expansion.

It has been already proposed that modest ring exchange $J_{r}>0$ on TL destroys the magnetic LRO and induces SL g.s. $[25,26]$, including the observation of several possible singlet excitations below the lowest triplet one. In Fig. 7 we present results for the HM on TL with $J_{r}>0$, Eq. (9), as obtained via FTLM on $N=28$ sites (smaller size due to more complex $H$ ). It is evident that $J_{r}>0$ steadily increases low- $T$ entropy $s(T)$ while increasing $\chi_{0}(T)$. The resulting $R(T)$ looses magnetic LRO character already for $J_{r} \geqslant 0.05$, being followed by an SL-like regime with vanishing $R_{0} \rightarrow 0$.

The same message follows from the consideration of lowest levels on a $N=28$ lattice, presented in Fig. 5(c).
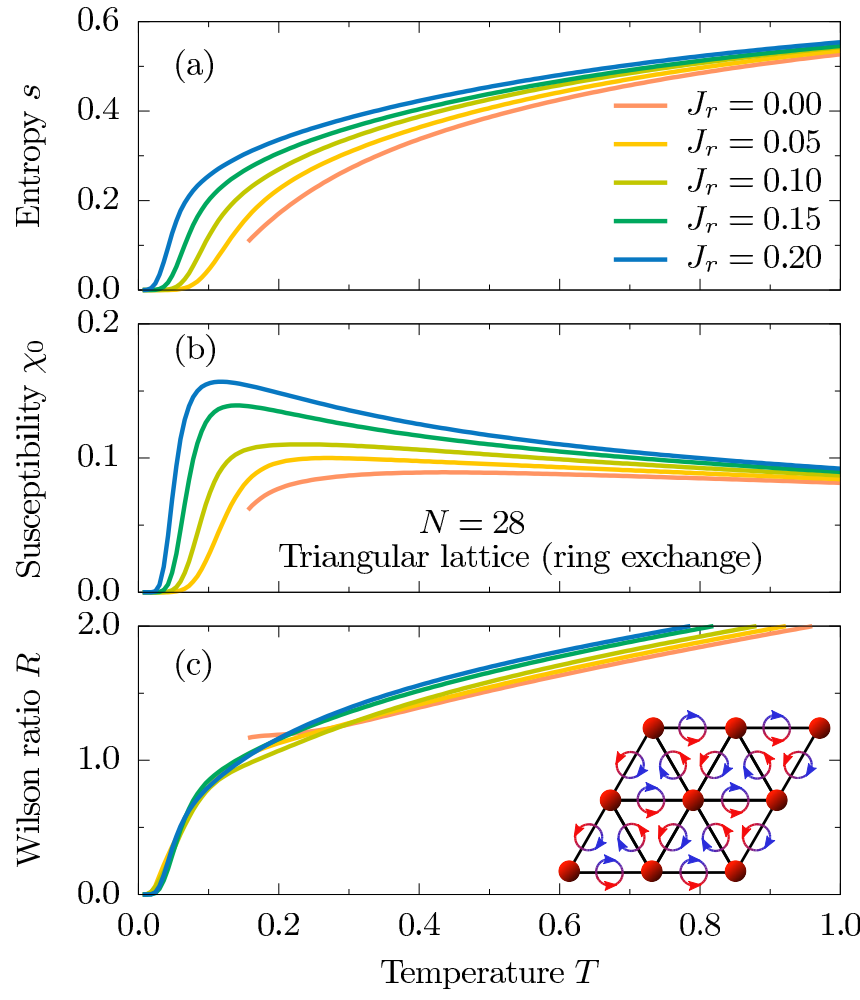

FIG. 7. $s(T), \chi_{0}(T)$, and $R(T)$ within HM on TL, including ring exchange, obtained via FTLM on $N=28$ sites for different $0 \leqslant J_{r} \leqslant 0.2$. The inset in (c) represents a sketch of the $J$ (solid line) connections and the ring exchange $J_{r}$ (circle) in TL.

Analogous to Figs. 5(a) and 5(b), there is a clear collapse of singlet levels $\epsilon_{s, 1-3}$ (here we employ a q-resolved code and cannot monitor all singlet excitations) below the triplet one $\epsilon_{t}$ for $J_{r}>0.1$. In the latter regime $\epsilon_{t}$ represents an already reasonable estimate of the limiting $N \rightarrow \infty$ triplet gap $\Delta_{t}>0$ [25], whereas to establish a proper singlet gap (the lowest singlet in $N \rightarrow \infty$ limit), $\Delta_{s}<\Delta_{t}$ requires more detailed finite-size analysis.

\section{D. $J_{1}-J_{2}$ Heisenberg model on square lattice}

Finally, we turn to the $J_{1}-J_{2} \mathrm{HM}$, Eq. (7), on SQL. The latter has been one of first considered for the possible (plaquette) VB solid g.s. at intermediate $J_{2} \sim 0.5[11,12,16,74,75]$, but also for the SL g.s. [13-15,17,18]. Results for corresponding thermodynamic quantities presented in Figs. 8(c) and 8(d) are consistent with the diverging $R_{0} \rightarrow \infty$, indicating magnetic LRO outside a quite narrow parameter regime, i.e., outside $0.5 \leqslant J_{2} \leqslant 0.6$. In the latter regime we again find substantial entropy $s(T \ll 1)$ and consequently $R_{0} \rightarrow 0$, whereby for $J_{2} \sim 0.6$ there are already some indications for possible degeneracy $s_{0}>0$ which could be in favor of broken translational symmetry, e.g., a plaquette VB solid [11,12,16,74,75].

Caveats for the SL interpretation emerge also when considering the excitation evolution vs $J_{2}$ [see Fig. 5(d)], as obtained from ED results on an $N=36$ cluster. For given system size, the singlet levels reveal $\epsilon_{s, 1-6}<\epsilon_{t}$ only in a very narrow regime $0.55<J_{2}<0.62$. Even then, higher singlets (apart from $\epsilon_{s, 1}$ ) are not well below $\epsilon_{t}$. Consistent with previous 

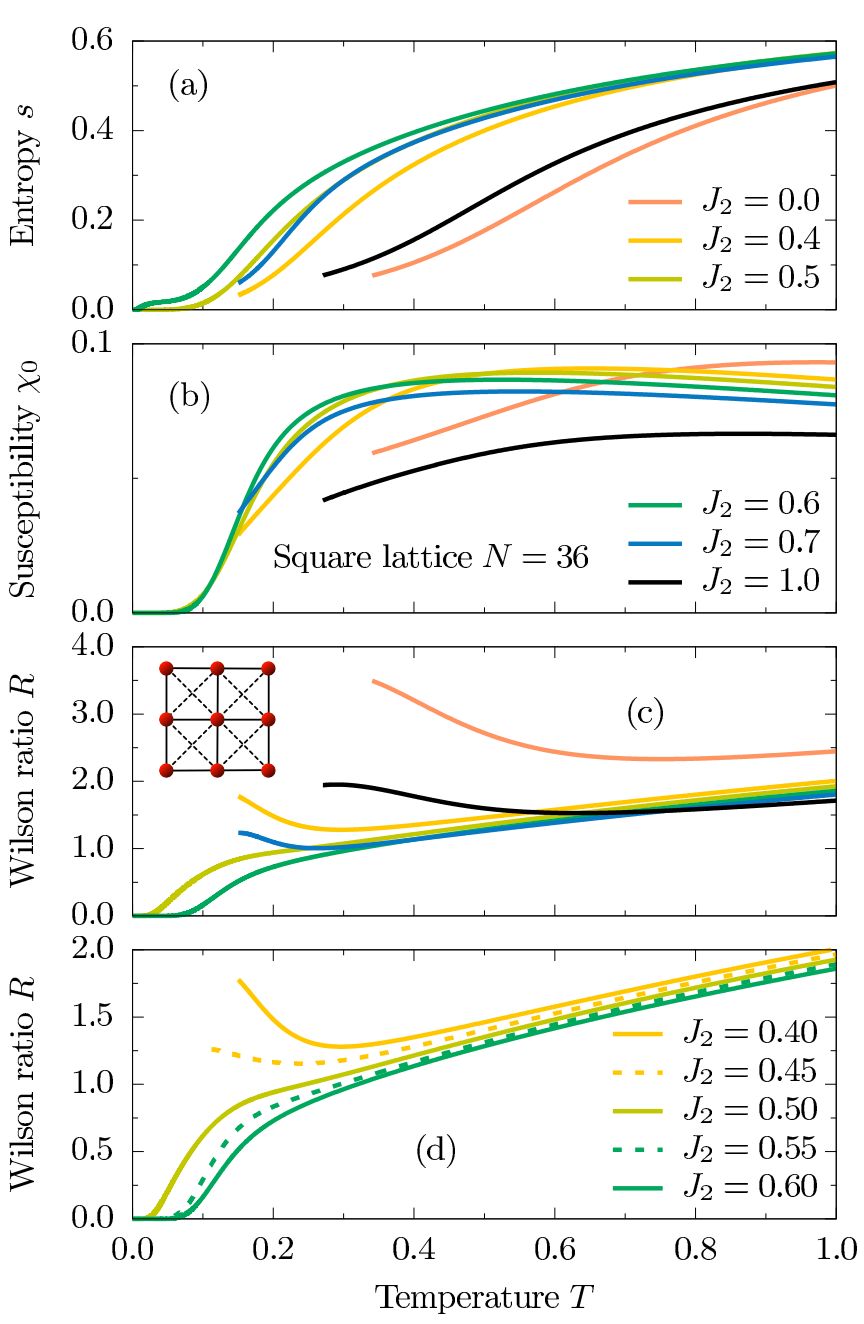

FIG. 8. (a) $s(T)$, (b) $\chi_{0}(T)$, and (c) $R(T)$ within $J_{1}-J_{2} \mathrm{HM}$ on $\mathrm{SQL}$, obtained via FTLM on $N=36$ sites for different $0 \leqslant J_{2} \leqslant 1.0$. In (d) $R(T)$ results are presented within the expanded intermediate regime $0.4 \leqslant J_{2} \leqslant 0.6$.

works [11-18], the level scheme indicates a change of the g.s. character for $J_{2}>0.6$. As a consequence, the SL in the intermediate regime, and even more on the singlet-dominated regime, is less conclusive, and other options [17,75] also have to be considered.

\section{CONCLUSIONS}

The thermodynamic quantities, entropy density $s(T)$ (together with directly related specific heat $C_{V}(T)=T d s / d T$, not presented in this paper), uniform susceptibility $\chi_{0}(T)$, and consequently $T$-dependent Wilson ratio $R(T)$, offer another view on properties of frustrated spin models. We considered here prototype 2D isotropic $S=1 / 2 \mathrm{HM}$, which are at least in some parameter regimes best candidates for the SL g.s. For comparison, we investigate in the same manner also the simplest 1D HM which can serve as reference for some concepts and scenarios.

$R(T)$, in particular its low- $T$ variation, is the quantity which differentiates between different scenarios. Whereas 2D systems with magnetic LRO can be monitored via $R_{0} \rightarrow \infty$, we are more interested in the SL regimes with g.s. without magnetic LRO and even without any broken translational symmetry, which could be classified as VB solid. As a prototype case we present results for $1 \mathrm{D} J_{1}-J_{2} \mathrm{HM}$, which does not have magnetic LRO but offers already two firm scenarios: (a) the gapless regime for $J_{2}<J_{2}^{*}$ with spinons (or 1D SFS) as elementary excitations, and consequently finite $R_{0}=R(T \rightarrow$ $0) \sim 2$ (for $J_{2} \sim 0$ ), and (b) a gapped regime for $J_{2}>J_{2}^{*}$ with dimerized g.s. (being the simplest $1 \mathrm{D}$ form of VB solid) apparently also with $R_{0}=0$, although not yet a fully resolved variation $R(T \rightarrow 0)$.

SL regimes in considered 2D frustrated isotropic $S=1 / 2$ $\mathrm{HM}$ are in our study located via enhanced low- $T$ entropy $s(T)$ and gapped (or at least reduced) $\chi_{0}(T)$, resulting in vanishing $R_{0}=0$. Similar information and criterion (although less well defined) emerges from the excitation spectra when differentiating singlet and triplet (or even higher $S_{\text {tot }}>1$ ) excitations over the $S_{\text {tot }}=0$ g.s. Most evident cases for such VB (dimer) liquid scenarios appear within the KL around $J_{2} \sim 0$. An analogous, although somewhat less pronounced, case is obtained within HM on TL with ring exchange $J_{r}>$ 0.1 and for the $J_{1}-J_{2} \mathrm{HM}$ on TL in the intermediate regime $0.1<J_{2}<0.17$. For such systems the level evolution as well as $R(T)$ reveal a massive density of singlet states below the lowest triplet excitation. On the other hand, the situation in the $\mathrm{HM}$ on SQL in the narrow regime $J_{2} \sim 0.6$ is less clear-cut in this respect, since singlets are not well below the lowest triplet.

Vanishing $R_{0}$ does not support the scenario of SL with large (or even Dirac-cone) spinon Fermi surfaces, which would require finite $R_{0}>0$ (as in 1D HM), although our finite-size studies should be interpreted with care and cannot give a final answer to this problem. Still, the emergent scenario of VB liquid should be critically faced with the possibility of VB solid. In the latter case the g.s. should be (due to broken translational symmetry) degenerate with finite $N_{d}>1$ (in the thermodynamic limit $N \rightarrow \infty$ ). We find clear numerical evidence for $N_{d}>1$ within the $J_{1}-J_{2} \mathrm{HM}$ on TL for $J_{2}>0.2$, but in this case it is consistent with the striped magnetic LRO. Some indication for $N_{d}>1$ appears also for the SQL at $J_{2} \sim 0.6$, which might support the existence of a (plaquette) VB solid [14,17] in this regime instead of SL (without broken translational symmetry). Results in the (presumable) SL regimes also indicate finite triplet gaps $\Delta_{t}>0$, while singlet gaps are either finite $\Delta_{S}>0$ or vanishing $\Delta_{S} \sim 0$ (for the $J_{2} \sim 0 \mathrm{KL}$ model), but evidently $\Delta_{s}<\Delta_{t}$. To establish (or exclude) possible $N_{d}>1$ and to determine $\Delta_{s}>0$ beyond doubt still requires further studies.

Finally, it should be stressed that the evaluated thermodynamic quantities are (at least in principle) measurable in experimental realizations of SL materials. $s(T)$ is accessible via measured magnetic specific heat $C_{V}(T)$ and uniform susceptibility $\chi_{0}(T)$ via macroscopic d.c. or/and Knight-shift measurement. Since known SL materials are characterized by modest exchange $J$, properties can be measured in the wide range $T \lesssim J$. This offers the possibility of critical comparison with model results, whereby a considered isotropic HM might still miss some ingredients relevant for the low- $T$ behavior, in particular, the Dzyaloshinskii-Moriya interaction, the disorder influence, and the interlayer coupling. 


\section{ACKNOWLEDGMENTS}

P.P. is supported by the P1-0044 program and Project No. N1-0088 of the Slovenian Research Agency. K.M. and T.T. are supported by MEXT, Japan, as a social and scientific priority issue (creation of new functional devices and highperformance materials to support next-generation industries) to be tackled by using a post-K computer. T.T. is also

[1] P. A. Lee, An end to the drought of quantum spin liquids, Science 321, 1306 (2008).

[2] L. Balents, Spin liquids in frustrated magnets, Nature (London) 464, 199 (2010).

[3] L. Savary and L. Balents, Quantum spin liquids: A review, Rep. Prog. Phys. 80, 016502 (2017).

[4] F. Mila, Low-Energy Sector of the Kagome Antiferromagnet, Phys. Rev. Lett. 81, 2356 (1998).

[5] R. Budnik and A. Auerbach, Low-Energy Singlets in the Heisenberg Antiferromagnet on the Kagome Lattice, Phys. Rev. Lett. 93, 187205 (2004).

[6] R. R. P. Singh and D. A. Huse, Ground state of the spin-1/2 kagome-lattice Heisenberg antiferromagnet, Phys. Rev. B 76, 180407(R) (2007).

[7] S. Yan, D. A. Huse, and S. R. White, Spin-liquid ground state of the $S=1 / 2$ kagome Heisenberg antiferromagnet, Science 322, 1173 (2008).

[8] A. M. Läuchli, J. Sudan, and E. S. Sørensen, Ground-state energy and spin gap of spin-1/2 kagomé-Heisenberg antiferromagnetic clusters: Large-scale exact diagonalization results, Phys. Rev. B 83, 212401 (2011).

[9] Y. Iqbal, F. Becca, and D. Poilblanc, Valence-bond crystal in the extended kagome spin-1/2 quantum Heisenberg antiferromagnet: A variational Monte Carlo approach, Phys. Rev. B 83, 100404(R) (2011).

[10] S. Depenbrock, I. P. McCulloch, and U. Schollwöck, Nature of the Spin-Liquid Ground State of the $s=1 / 2$ Heisenberg Model on the Kagome Lattice, Phys. Rev. Lett. 109, 067201 (2012).

[11] L. Capriotti and S. Sorella, Spontaneous Plaquette Dimerization in the J1-J2 Heisenberg Model, Phys. Rev. Lett. 84, 3173 (2000).

[12] M. Mambrini, A. Läuchli, D. Poilblanc, and F. Mila, Plaquette valence-bond crystal in the frustrated Heisenberg quantum antiferromagnet on the square lattice, Phys. Rev. B 74, 144422 (2006).

[13] H. C. Jiang, H. Yao, and L. Balents, Spin liquid ground state of the spin-12 square J1-J2 Heisenberg model, Phys. Rev. B 86, 024424 (2012).

[14] S. S. Gong, W. Zhu, D. N. Sheng, O. I. Motrunich, and M. P. A. Fisher, Plaquette Ordered Phase and Quantum Phase Diagram in the Spin- 12 J1-J2 Square Heisenberg Model, Phys. Rev. Lett. 113, 027201 (2014).

[15] S. Morita, R. Kaneko, and M. Imada, Quantum spin liquid in spin 1/2 J1-J2 Heisenberg model on square lattice: Many-variable variational Monte Carlo study combined with quantum-number projections, J. Phys. Soc. Jpn. 84, 024720 (2015). supported by the JSPS KAKENHI (No. JP19H05825). The numerical calculation was partly carried out at the facilities of the Supercomputer Center, the Institute for Solid State Physics, the University of Tokyo, at the Yukawa Institute Computer Facility, Kyoto University, and at the Wroclaw Centre for Networking and Supercomputing. J.H. acknowledges grant support by the Polish National Agency of Academic Exchange (NAWA) under Contract No. PPN/PPO/2018/1/00035.
[16] K. Morita and N. Shibata, Field-induced quantum phase transitions in $s=1 / 2 \mathrm{j} 1-\mathrm{j} 2$ Heisenberg model on square lattice, $\mathrm{J}$. Phys. Soc. Jpn. 85, 094708 (2016).

[17] L. Wang and A. W. Sandvik, Critical Level Crossings and Gapless Spin Liquid in the Square-Lattice Spin- 1 /2 J1-J2 Heisenberg Antiferromagnet, Phys. Rev. Lett. 121, 107202 (2018).

[18] W.-Y. Liu, S. Dong, C. Wang, Y. Han, H. An, G.-C. Guo, and L. He, Gapless spin liquid ground state of the spin-1/2 j1-j2 Heisenberg model on square lattices, Phys. Rev. B 98, 241109(R) (2018).

[19] R. Kaneko, S. Morita, and M. Imada, Gapless spin-liquid phase in an extended spin-1/2 triangular Heisenberg model, J. Phys. Soc. Jpn. 83, 093707 (2014).

[20] Z. Zhu and S. R. White, Spin liquid phase of the $S=1 / 2$ J1-J2 Heisenberg model on the triangular lattice, Phys. Rev. B 92, 041105(R) (2015).

[21] W. J. Hu, S. S. Gong, W. Zhu, and D. N. Sheng, Competing spin-liquid states in the spin-12 Heisenberg model on the triangular lattice, Phys. Rev. B 92, 140403(R) (2015).

[22] Y. Iqbal, W.-J. Hu, R. Thomale, D. Poilblanc, and F. Becca, Spin liquid nature in the Heisenberg, Phys. Rev. B 93, 144411 (2016).

[23] A. Wietek and A. M. Läuchli, Chiral spin liquid and quantum criticality in extended $s=\frac{1}{2}$ Heisenberg models on the triangular lattice, Phys. Rev. B 95, 035141 (2017).

[24] P. Prelovšek and J. Kokalj, Finite-temperature properties of the extended Heisenberg model on a triangular lattice, Phys. Rev. B 98, 035107 (2018).

[25] G. Misguich, C. Lhuillier, B. Bernu, and C. Waldtmann, Spinliquid phase of the multiple-spin exchange Hamiltonian on the triangular lattice, Phys. Rev. B 60, 1064 (1999).

[26] Olexei I. Motrunich, Variational study of triangular lattice spin-1/2 model with ring exchanges and spin liquid state in $\kappa-(\mathrm{ET})_{2} \mathrm{Cu}_{2}(\mathrm{CN})_{3}$, Phys. Rev. B 72, 045105 (2005).

[27] P. Mendels, F. Bert, M. A. de Vries, A. Olariu, A. Harrison, F. Duc, J. C. Trombe, J. S. Lord, A. Amato, and C. Baines, Quantum Magnetism in the Paratacamite Family: Towards an Ideal Kagomé Lattice, Phys. Rev. Lett. 98, 077204 (2007).

[28] A. Olariu, P. Mendels, F. Bert, F. Duc, J. C. Trombe, M. A. de Vries, and A. Harrison, ${ }^{17} \mathrm{O}$ NMR Study of the Intrinsic Magnetic Susceptibility and Spin Dynamics of the Quantum Kagome Antiferromagnet $\mathrm{ZnCu}_{3}(\mathrm{OH})_{6} \mathrm{Cl}_{2}$, Phys. Rev. Lett. 100, 087202 (2008).

[29] T. H. Han, J. S. Helton, S. Chu, D. G. Nocera, J. A. RodriguezRivera, C. Broholm, and Y. S. Lee, Fractionalized excitations in the spin-liquid state of a kagome-lattice antiferromagnet, Nature (London) 492, 406 (2012). 
[30] M. Fu, T. Imai, T.-H. Han, and Y. S. Lee, Evidence for a gapped spin-liquid ground state in a kagome Heisenberg antiferromagnet, Science 350, 655 (2015).

[31] M. R. Norman, Colloquium: Herbertsmithite and the search for the quantum spin liquid, Rev. Mod. Phys. 88, 041002 (2016).

[32] Z. Hiroi, M. Hanawa, N. Kobayashi, M. Nohara, H. Takagi, Y. Kato, and M. Takigawa, Spin-1/2 kagome-like lattice in volborthite $\mathrm{Cu}_{3} \mathrm{~V}_{2} \mathrm{O}_{7}(\mathrm{OH})_{2} \cdot 2 \mathrm{H}_{2} \mathrm{O}$, J. Phys. Soc. Jpn. 70, 3377 (2001).

[33] B. Fåk, E. Kermarrec, L. Messio, B. Bernu, C. Lhuillier, F. Bert, P. Mendels, B. Koteswararao, F. Bouquet, J. Ollivier, A. D. Hillier, A. Amato, R. H. Colman, and A. S. Wills, Kapellasite: A Kagome Quantum Spin Liquid with Competing Interactions, Phys. Rev. Lett. 109, 037208 (2012).

[34] L. Yuesheng, B. Pan, S. Li, W. Tong, Ling. L., Z. Yang, J. Wang, Z. Chen, Z. Wu, and Q. Zhang, Gapless quantum spin liquid in the $\mathrm{S}=1 / 2$ anisotropic kagome antiferromagnet $\mathrm{ZnCu}_{3}(\mathrm{OH}) 6 \mathrm{SO}_{4}$, New J. Phys. 16, 093011 (2014).

[35] M. Gomilšek, M. Klanjšek, M. Pregelj, F. C. Coomer, H. Luetkens, O. Zaharko, T. Fennell, Y. Li, Q. M. Zhang, and A. Zorko, Instabilities of spin-liquid states in a quantum kagome antiferromagnet, Phys. Rev. B 93, 060405(R) (2016).

[36] Z. Feng, Z. Li, X. Meng, W. Yi, Y. Wei, J. Zhang, Y. C. Wang, W. Jiang, Z. Liu, S. Li, F. Liu, J. Luo, S. Li, G. Q. Zheng, Z. Y. Meng, J. W. Mei, and Y. Shi, Gapped spin-1/2 spinon excitations in a new kagome quantum spin liquid compound $\mathrm{Cu}_{3} \mathrm{Zn}(\mathrm{OH}) 6 \mathrm{FBr}$, Chinese Phys. Lett. 34, 077502 (2017)

[37] A. Zorko, M. Pregelj, M. Klanjšek, M. Gomilšek, Z. Jagličić, J. S. Lord, J. A. T. Verezhak, T. Shang, W. Sun, and J.-X. Mi, Coexistence of magnetic order and persistent spin dynamics in a quantum kagome antiferromagnet with no intersite mixing, Phys. Rev. B 99, 214441 (2019).

[38] Y. Shimizu, K. Miyagawa, K. Kanoda, M. Maesato, and G. Saito, Spin Liquid State in an Organic Mott Insulator with a Triangular Lattice, Phys. Rev. Lett. 91, 107001 (2003).

[39] Y. Shimizu, K. Miyagawa, K. Kanoda, M. Maesato, and G. Saito, Emergence of inhomogeneous moments from spin liquid in the triangular-lattice Mott insulator $\kappa-(\mathrm{ET})_{2} \mathrm{cu}_{2}(\mathrm{CN})_{3}$, Phys. Rev. B 73, 140407(R) (2006).

[40] T. Itou, A. Oyamada, S. Maegawa, and R. Kato, Instability of a quantum spin liquid in an organic triangular-lattice antiferromagnet, Nat. Phys. 6, 673 (2010).

[41] Y. Zhou, K. Kanoda, and T. K. Ng, Quantum spin liquid states, Rev. Mod. Phys. 89, 025003 (2017).

[42] M. Klanjšek, A. Zorko, R. Žitko, J. Mravlje, Z Jagličić, P. K. Biswas, P. Prelovšek, D. Mihailovic, and D. Arčon, A hightemperature quantum spin liquid with polaron spins, Nat. Phys. 13, 1130 (2017).

[43] M. Kratochvilova, A. D. Hillier, A. R. Wildes, L. Wang, S.-W. Cheong, and J.-G. Park, The low-temperature highly correlated quantum phase in the charge-density-wave 1T-TaS2 compound, Quantum Mat. 2, 42 (2017).

[44] K. T. Law and P. A. Lee, 1T-TaS2 as a quantum spin liquid, Proc. Natl. Acad. Sci. U.S.A. 114, 6996 (2017).

[45] W.-Y. He, X. Y. Xu, G. Chen, K. T. Law, and P. A. Lee, Spinon Fermi Surface in a Cluster Mott Insulator Model on a Triangular Lattice and Possible Application to 1t-Tas2, Phys. Rev. Lett. 121, 046401 (2018).
[46] M. Rigol, T. Bryant, and R. R. P. Singh, Numerical linkedcluster algorithms, I. Spin systems on square, triangular, and kagome lattices, Phys. Rev. E 75, 061118 (2007).

[47] O. Cépas, C. M. Fong, P. W. Leung, and C. Lhuillier, Quantum phase transition induced by Dzyaloshinskii-Moriya interactions in the kagome antiferromagnet, Phys. Rev. B 78, 140405(R) (2008).

[48] A. Zorko, S. Nellutla, J. van Tol, L. C. Brunel, F. Bert, F. Duc, J. C. Trombe, M. A. de Vries, A. Harrison, and P. Mendels, Dzyaloshinsky-Moriya Anisotropy in the Spin-1/2 Kagome Compound $\mathrm{ZnCu}_{3}(\mathrm{OH}) 6 \mathrm{Cl}_{2}$, Phys. Rev. Lett. 101, 026405 (2008).

[49] H. Kawamura and K. Uematsu, Nature of the randomnessinduced quantum spin liquids in two dimensions, J. Phys.: Condens. Matter 31, 504003 (2019).

[50] R. R. P. Singh and D. A. Huse, Triplet and singlet excitations in the valence bond crystal phase of the kagome lattice Heisenberg model, Phys. Rev. B 77, 144415 (2008).

[51] A. M. Läuchli, J. Sudan, and R. Moessner, $S=1 / 2$ kagome Heisenberg antiferromagnet revisited, Phys. Rev. B 100, 155142 (2019).

[52] Y. Iqbal, F. Becca, S. Sorella, and D. Poilblanc, Gapless spinliquid phase in the kagome spin- $\frac{1}{2}$ Heisenberg antiferromagnet, Phys. Rev. B 87, 060405(R) (2013).

[53] Y.-C. He, M. P. Zaletel, M. Oshikawa, and F. Pollmann, Signatures of Dirac Cones in a DMRG Study of the Kagome Heisenberg Model, Phys. Rev. X 7, 031020 (2017).

[54] P. Prelovšek and J. Kokalj, Similarity of thermodynamic properties of Heisenberg model on triangular and kagome lattices, Phys. Rev. B 101, 075105 (2020).

[55] G. Misguich and P. Sindzingre, Magnetic susceptibility and specific heat of the spin-1/2 Heisenberg model on the kagome lattice and experimental data on $\mathrm{ZnCu}_{3}(\mathrm{OH}) 6 \mathrm{Cl}_{2}$, Eur. Phys. J. B 59, 305 (2007).

[56] J. Schnack, J. Schulenburg, and J. Richter, Magnetism of the $n=42$ kagome lattice antiferromagnet, Phys. Rev. B 98, 094423 (2018).

[57] Ch. Waldtmann, H. U. Everts, B. Bernu, C. Lhuillier, P. Sindzingre, P. Lecheminant, and L. Pierre, First excitations of the spin 1/2 Heisenberg antiferromagnet on the kagomé lattice, Eur. Phys. J. B 2, 501 (1998).

[58] J. Jaklič and P. Prelovšek, Finite-temperature properties of doped antiferromagnets, Adv. Phys. 49, 1 (2000).

[59] K. Ninios, Tao Hong, T. Manabe, C. Hotta, S. N. Herringer, M. M. Turnbull, C. P. Landee, Y. Takano, and H. B. Chan, Wilson Ratio of a Tomonaga-Luttinger Liquid in a Spin-1/2 Heisenberg Ladder, Phys. Rev. Lett. 108, 097201 (2012).

[60] E. Manousakis, The spin-1/2 Heisenberg antiferromagnet on a square lattice and its application to the cuprous oxides, Rev. Mod. Phys. 63, 1 (1991).

[61] D. C. Johnston, R. K. Kremer, M. Troyer, X. Wang, A. Klümper, S. L. Bud'ko, A. F. Panchula, and P. C. Canfield, Thermodynamics of spin $s=1 / 2$ antiferromagnetic uniform and alternating-exchange Heisenberg chains, Phys. Rev. B 61, 9558 (2000).

[62] J. Jaklič and P. Prelovšek, Finite-temperature conductivity in the planar t-j model, Phys. Rev. B 50, 7129 (1994).

[63] P. Prelovšek and J. Bonča, Ground state and finite temperature Lanczos methods, in Strongly Correlated Systems - Numerical 
Methods, edited by A. Avella and F. Mancini (Springer, Berlin, 2013).

[64] K. Morita and T. Tohyama, Finite-temperature properties of the Kitaev-Heisenberg models on kagome and triangular lattices studied by improved finite-temperature Lanczos methods, Phys. Rev. Res. 2, 013205 (2020).

[65] S. R. White and I. Affleck, Dimerization and incommensurate spiral spin correlations in the zigzag spin chain: Analogies to the Kondo lattice, Phys. Rev. B 54, 9862 (1996).

[66] H. J. Liao, Z. Y. Xie, J. Chen, Z. Y. Liu, H. D. Xie, R. Z. Huang, B. Normand, and T. Xiang, Gapless Spin-Liquid Ground State in the $s=1 / 2$ Kagome Antiferromagnet, Phys. Rev. Lett. 118, 137202 (2017).

[67] F. Kolley, S. Depenbrock, I. P. McCulloch, U. Schollwöck, and V. Alba, Phase diagram of the $J_{1}-J_{2}$ Heisenberg model on the kagome lattice, Phys. Rev. B 91, 104418 (2015).

[68] V. Subrahmanyam, Block spins and chirality in the frustrated Heisenberg model on kagome and triangular lattices, Phys. Rev. B 52, 1133 (1995).

[69] B. Bernu, P. Lecheminant, C. Lhuillier, and L. Pierre, Exact spectra, spin susceptibilities, and order parameter of the quan- tum Heisenberg antiferromagnet on the triangular lattice, Phys. Rev. B 50, 10048 (1994).

[70] L. Capriotti, A. E. Trumper, and S. Sorella, Long-Range Néel Order in the Triangular Heisenberg Model, Phys. Rev. Lett. 82, 3899 (1999).

[71] S. R. White and A. L. Chernyshev, Néel Order in Square and Triangular Lattice Heisenberg Models, Phys. Rev. Lett. 99, 127004 (2007).

[72] H. Y. Yang, A. M. Lauchli, F. Mila, and K. P. Schmidt, Effective Spin Model for the Spin-Liquid Phase of the Hubbard Model on the Triangular Lattice, Phys. Rev. Lett. 105, 267204 (2010).

[73] Y. Nakamura, N. Yoneyama, T. Sasaki, T. Tohyama, A. Nakamura, and H. Kishida, Magnetic Raman scattering study of spin frustrated systems, $\kappa$-(BEDT-TTF)2X, J. Phys. Soc. Jpn. 83, 074708 (2014).

[74] R. L. Doretto, Plaquette valence-bond solid in the square-lattice $J_{1}-J_{2}$ antiferromagnet Heisenberg model: A bond operator approach, Phys. Rev. B 89, 104415 (2014).

[75] B. Zhao, J. Takahashi, and A. W. Sandvik, Comment on gapless spin liquid ground state of the spin- $1 / 2 J_{1}-J_{2}$ Heisenberg model on square lattices, arXiv:1909.12788. 